\title{
Lombardie, la région italienne la plus touchée par la Covid-19
}

Analyse des aspects socio-territoriaux du foyer de la Val Seriana

\section{Elisa Consolandi}

\section{OpenEdition}

\section{Journals}

Édition électronique

URL : http://journals.openedition.org/rfst/856

DOI : $10.4000 /$ rfst.856

ISSN : 2492-3672

Éditeur

Espaces et SOciétés (UMR 6590)

Référence électronique

Elisa Consolandi, «Lombardie, la région italienne la plus touchée par la Covid-19 », Revue francophone sur la santé et les territoires [En ligne], Pandémie, crises et perspectives : lectures territoriales de la Covid-19, mis en ligne le 18 mars 2021, consulté le 06 avril 2021. URL : http:// journals.openedition.org/rfst/856 ; DOI : https://doi.org/10.4000/rfst.856

Ce document a été généré automatiquement le 6 avril 2021.

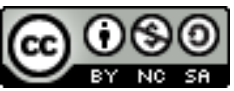

La Revue francophone sur la santé et les territoires est mise à disposition selon les termes de la Licence Creative Commons Attribution - Pas d'Utilisation Commerciale - Partage dans les Mêmes Conditions 4.0 International. 


\title{
Lombardie, la région italienne la plus touchée par la Covid-19
}

Analyse des aspects socio-territoriaux du foyer de la Val Seriana

\author{
Elisa Consolandi
}

\section{Préambule}

1 L'Italie a été le premier pays européen frappé par la Covid-19, et plus précisément ses régions septentrionales, qui ont été les plus lourdement touchées par le virus et ses conséquences les plus graves. La Lombardie en particulier est la région dans laquelle on a enregistré à la fois le plus grand nombre de cas et le plus grand nombre de décès. Les premiers foyers ${ }^{1}$ de la maladie ont été localisés dans la partie orientale de la région, à savoir dans la province de Lodi et dans la Val Seriana (province de Bergame), où a également été enregistré le nombre de cas le plus élevé rapporté à la population.

2 Cette étude, qui s'inscrit dans le cadre de travaux de recherche menés par le Centro Studi sul Territorio de l'Université de Bergame $^{2}$ et fondés sur une approche cartographique, a pour objet d'illustrer les caractéristiques de l'épidémie dans la région lombarde, en les mettant en relation avec certains aspects socio-territoriaux ${ }^{3}$ afin d'examiner l'hypothèse d'un lien de causalité entre la complexité des structures résidentielles et fonctionnelles de la région et la gravité de la contamination.

\section{Mobilité pendulaire, urbanité et propagation du virus}

La recherche ${ }^{4}$ part du principe que le mode de vie, dans le monde contemporain, est mobile et urbanisé (Casti, 2020a), se traduisant par un entrelacs de nœuds et connexions dans lequel l'habitat local n'est plus seulement déterminé par la capacité d'auto-organisation des sujets d'un territoire, mais inclut également l'interaction avec les réseaux mondiaux (Lévy, 2008). La méthodologie de l'analyse conduite ici est fortement liée à cette vision et repose sur une approche comparative dans une perspective spatio-temporelle s'appuyant sur la cartographie, qui permet de visualiser, 
d'analyser et enfin d'interpréter les données. Ainsi, la cartographie réflexive (Casti, 2013) apparaît, à travers le croisement des données relatives aux cas de contamination et des données socio-territoriales, comme un instrument de représentation de nature à illustrer la complexité du phénomène épidémique. Les cartes qui vont être proposées et analysées plus loin ne portent donc pas exclusivement sur le suivi du nombre de cas en Italie mais dessinent sa séquence spatio-temporelle en présentant les aspects physiques et sociaux comme des causes possibles.

4 Par conséquent, en se basant sur ce critère, une attention particulière a été portée à la mobilité des habitants en fonction du type d'habitat. Sur le premier aspect, l'accent a été mis sur les déplacements quotidiens, autrement dit la mobilité pendulaire, considérée comme un indicateur de déplacement des individus entre différents territoires. En effet, la mobilité pendulaire pour motifs scolaires ou professionnels favorise l'intensification de la diffusion de la Covid-19 en raison des rassemblements qu'elle provoque à certains moments de la journée dans les moyens de transport publics ${ }^{5}$. En référence au second point, il faut souligner que la Lombardie se caractérise par une conurbation polycentrique constituée par la zone métropolitaine de Milan et par un ensemble de petites et moyennes villes qui l'entourent, constituant un modèle unique d'habitation. Ces polarités urbaines prennent un relief particulier en période de pandémie, à la fois en raison de leur densité de population, et donc du risque potentiel de diffusion du virus associé à la proximité, et parce qu'elles sont caractérisées par des déplacements de type rhizomatique, qui induisent une diffusion par réticularitét. En conséquence, l'intensité des interactions sociales qui caractérise la Lombardie, particulièrement dans les zones fortement urbanisées ${ }^{7}$, est un élément qui doit être pris en compte dans la mesure où, rapportée à la densité de population et au fonctionnement d'une société urbaine, c'est un facteur déterminant de la complexité relationnelle ${ }^{8}$.

5 À l'aide de certaines données relatives à la mobilité pendulaire et aux informations liées à la répartition de la population, nous allons maintenant décrire la diffusion du virus en Lombardie, en nous intéressant à la province de Bergame, et plus particulièrement au foyer de la Val Seriana, en raison de l'impact qu'il a eu sur la vitesse de propagation de la maladie. Les éléments cartographiques proposés, qui font office d'opérateurs symboliques', ont pour objectif de représenter un phénomène spécifique et, en même temps, d'en décrypter le sens social sous l'angle de la réflexivité (Casti, 1998, 2013), afin de mieux approfondir le phénomène pandémique.

\section{Le territoire de la Lombardie : l'épicentre italien}

Pour mieux comprendre pourquoi l'infection par la Covid-19 a eu un tel impact sur le territoire de la Lombardie, il convient pour commencer de s'intéresser à la répartition et à la structure de la population des territoires majoritairement touchés par le virus, afin d'établir ensuite une analogie avec la diffusion et la gravité de l'infection ${ }^{10}$. 
Figure 1. Répartition et subdivision par groupe d'âge de la population en Lombardie

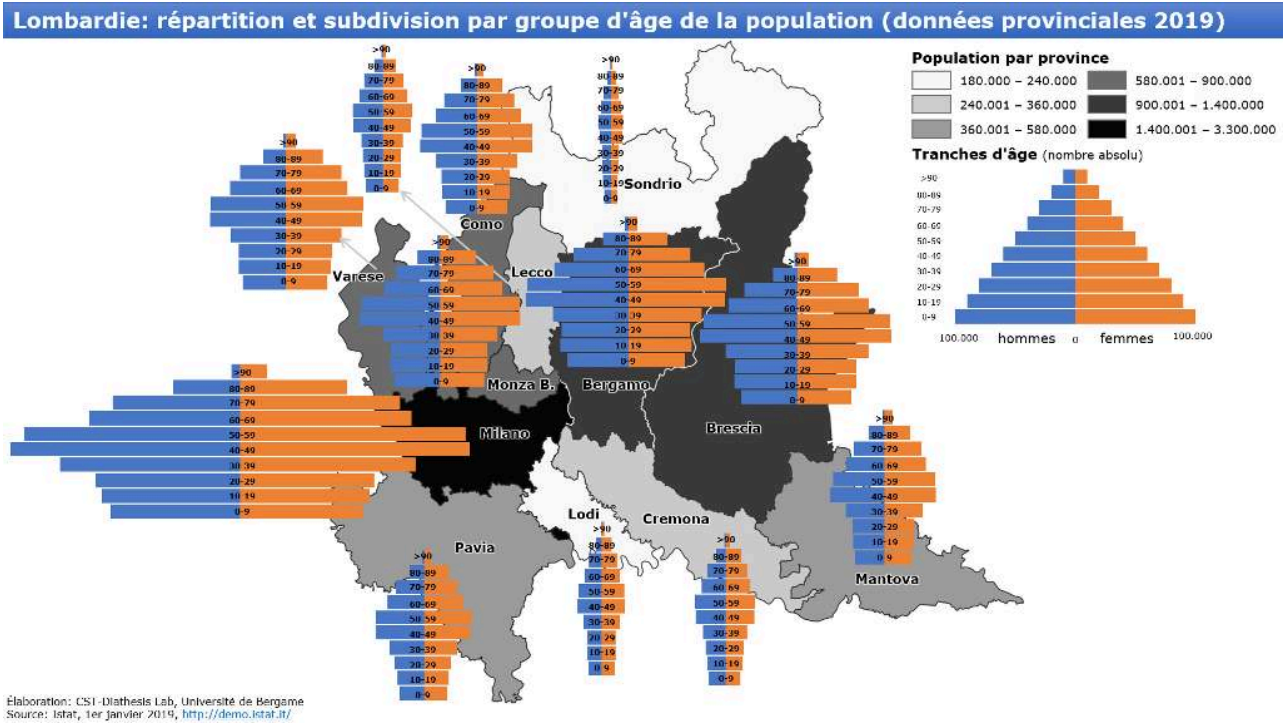

La représentation cartographique (Fig. 1) fait apparaître une grande diversité dans la répartition de la population des différentes provinces : la ville métropolitaine de Milan apparaît comme le territoire le plus densément peuplé, suivi des provinces de Bergame et de Brescia. À l'inverse, Lodi et Sondrio sont les villes qui comptent le plus petit nombre d'habitants. En ce qui concerne sa composition, la population présente en revanche le même profil que les autres régions d'Italie, à savoir une pyramide de forme inhabituelle: la tranche d'âge la plus nombreuse est celle qui regroupe les habitants entre 40 et 59 ans, tandis que les tranches d'âge inférieures affichent une nette contraction - signe de faible natalité - et les tranches supérieures suivent une évolution progressive d'ordre naturel, mais qui fait apparaître une espérance de vie élevée dans cette région. La subdivision hommes/femmes est elle aussi conforme à une tendance nationale, à savoir qu'il y a plus de femmes que d'hommes ${ }^{11}$.

Pour en venir à présent à l'étude de la diffusion des cas de contamination à la Covid-19 sur le territoire de la Lombardie, il convient de souligner qu'en l'espace de quelques semaines (à titre indicatif, du début mars à la fin avril 2020) la situation s'est nettement aggravée au niveau régional, en particulier dans les communes de la partie orientale de la Lombardie qui s'étend du sud au nord et dans laquelle on identifie clairement une «dorsale » de diffusion épidémique intense. C'est dans cette partie du territoire que sont apparus les premiers foyers de Covid-19, dans certaines communes des provinces de Lodi et de Bergame. Comme le montre la figure 2, on a observé dès le début une répartition différente des contaminations, qui sont représentées ici au moyen de techniques cartographiques spécifiques. En effet, la carte basée sur les données initiales de contamination - au 23 mars 2020 -, reproduit la région à la fois sur une base topographique de son territoire (a) et sur une base déformée par rapport au nombre d'habitants (b). L'effet obtenu par la cartographie est très parlant car sur la carte topographique seules les nuances de couleur démontrent les différences de répartition des contaminations. Inversement, la seconde carte $^{12}$ - où le pourcentage de contaminations est rapporté à la dilatation ou à la contraction des zones selon que le nombre d'habitants est élevé ou réduit - fait apparaître clairement que la région la plus touchée est la partie centre-orientale, qui s'avère aussi être moins peuplée que la zone métropolitaine de Milan. Il est en effet important de rappeler que ce n'est pas dans les 
grands centres urbains densément peuplés, comme Milan, Bergame ou Brescia, que l'on a observé tout d'abord le taux de contamination le plus élevé, mais dans les petites ou moyennes communes de la conurbation régionale, ce qui semble contredire la thèse selon laquelle la densité de population serait la cause de l'épidémie ${ }^{13}$.

Figure 2. Lombardie : distribution des cas de Covid-19 rapportés à la population résidente au 23 mars 2020.

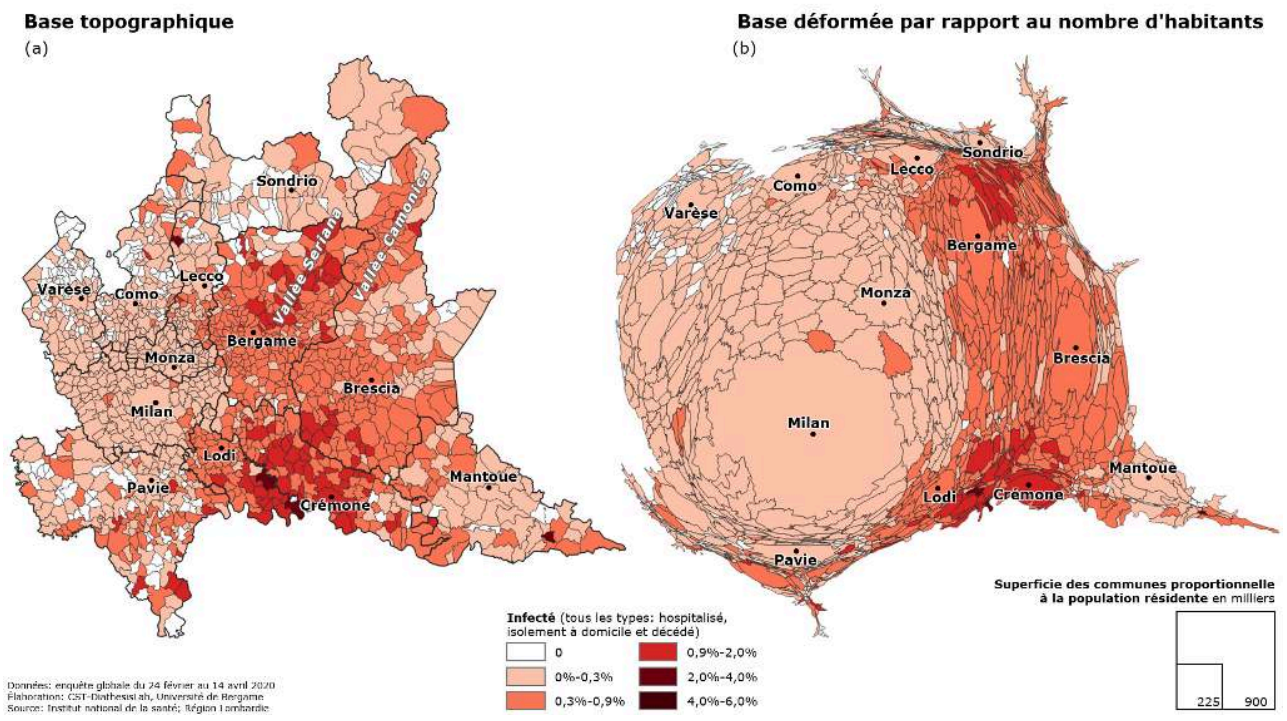

Inversement, il faut souligner que la diffusion du virus s'est ensuite intensifiée - c'est-àdire durant la phase de flambée épidémique -, essentiellement en zone urbaine. D'où l'hypothèse selon laquelle la propagation du virus serait liée, dans un premier temps, à une mécanique réticulaire, due aux contacts constants dans les aires périurbaines où sont apparus les premiers foyers. Dans un second temps, la transmission virologique semble avoir été favorisée par la proximité, se propageant plus facilement dans les espaces à densité élevée, comme le montre la figure $3^{14}$.

Figure 3. Zoom sur les foyers au 14 avril 2020

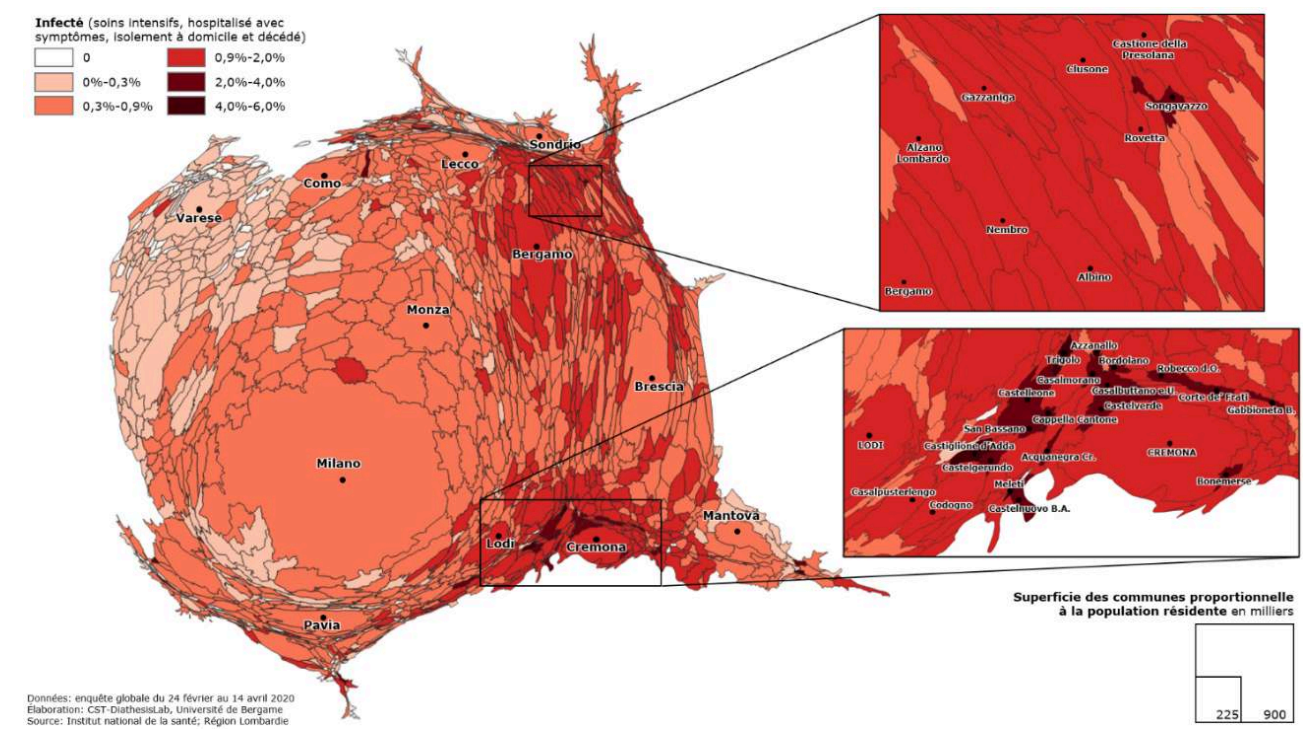


Quant à la gravité de la maladie et, par conséquent, aux chiffres négatifs plus marqués enregistrés en Lombardie, la forte mortalité recensée dans cette région ne peut être exclusivement expliquée par le nombre élevé de personnes âgées - des sujets très à risque et considérés comme la catégorie la plus fragile de la population. En effet, bien que leur population soit proportionnellement équivalente, les provinces ont enregistré d'importantes disparités au niveau du nombre de décès, pour preuve le taux de mortalité extrêmement élevé dans les provinces de Bergame, Crémone et Lodi ${ }^{15}$. Dans les territoires des provinces de Brescia, Pavie, Mantoue, Lecco et Sondrio, l'impact de la mortalité a également été plus élevé que dans d'autres régions d'Italie. Dans les provinces plus occidentales en revanche (dont l'aire métropolitaine de Milan), le taux de décès est inférieur et comparable à la moyenne italienne (Casti \& Adobati, 2020). L'explication de ces chiffres doit plutôt être cherchée ailleurs, par exemple dans l'inadaptation du système de santé et, plus généralement, dans la fragilité du système sanitaire et social, ce qui a contribué à la diffusion du virus dans les établissements d'hébergement pour personnes âgées et a fait apparaître les graves carences de gestion de ces structures, dues à la complexité et à la fragmentation extrêmes des compétences et des ressources entre acteurs institutionnels et non institutionnels (Ancidoni et.al., 2020; Berloto et.al., 2020) ${ }^{16}$.

11 Pour mieux comprendre cette thèse, aussi bien sous l'angle de la diffusion que celui de la gravité du virus, nous allons à présent passer à l'analyse d'un territoire fortement touché par la pandémie : la province de Bergame.

\section{Étude de cas : la province de Bergame}

12 La province de Bergame, au cœur de la vaste aire urbaine qui caractérise la plaine du Pô, est marquée par l'intensité des échanges et par la mobilité des habitants - tant au niveau local qu'à l'échelle nationale et internationale ${ }^{17}$. Le territoire de Bergame est considéré comme un important hub aéroportuaire pour le transport de passagers et de marchandises à finalité commerciale et industrielle. On est en présence d'un contexte mondialisé dynamique qui a consolidé, au fil du temps, un ensemble d'interactions et de liens socioculturels ${ }^{18}$.

13 Au regard de l'épidémie, Bergame fait partie des provinces italiennes dans lesquelles a été enregistré le plus grand nombre de cas de Covid-1919. La carte élaborée par interpolation des données relatives au nombre de contaminations ${ }^{20}$ avec les chiffres de la population résidente subdivisée par commune est représentée en anamorphose afin de faire mieux apparaître l'ampleur de l'épidémie. Ainsi, la métrique topographique a ici été abandonnée au profit de la métrique topographique, qui a pour but de décrypter le sens social du territoire et d'en illustrer de cette façon la complexité21 (Casti, 2013). La technique de représentation des communes ne repose donc pas sur leur superficie mais sur leur population résidente. Il est ainsi possible d'identifier à la fois la dimension des centres habités et la densité de population élevée de la plaine, car les territoires de montagne, dont les communes se situent au nord, au niveau des vallées bergamasques, présentent une superficie réduite en raison de leur faible population. Inversement, le pourcentage de personnes contaminées par le virus est représenté à travers les nuances de couleur initiales, ce qui nous permet de mesurer l'incidence de l'épidémie. 
Figure 4. Province de Bergame. Évolution du nombre de cas par rapport à la population résidente

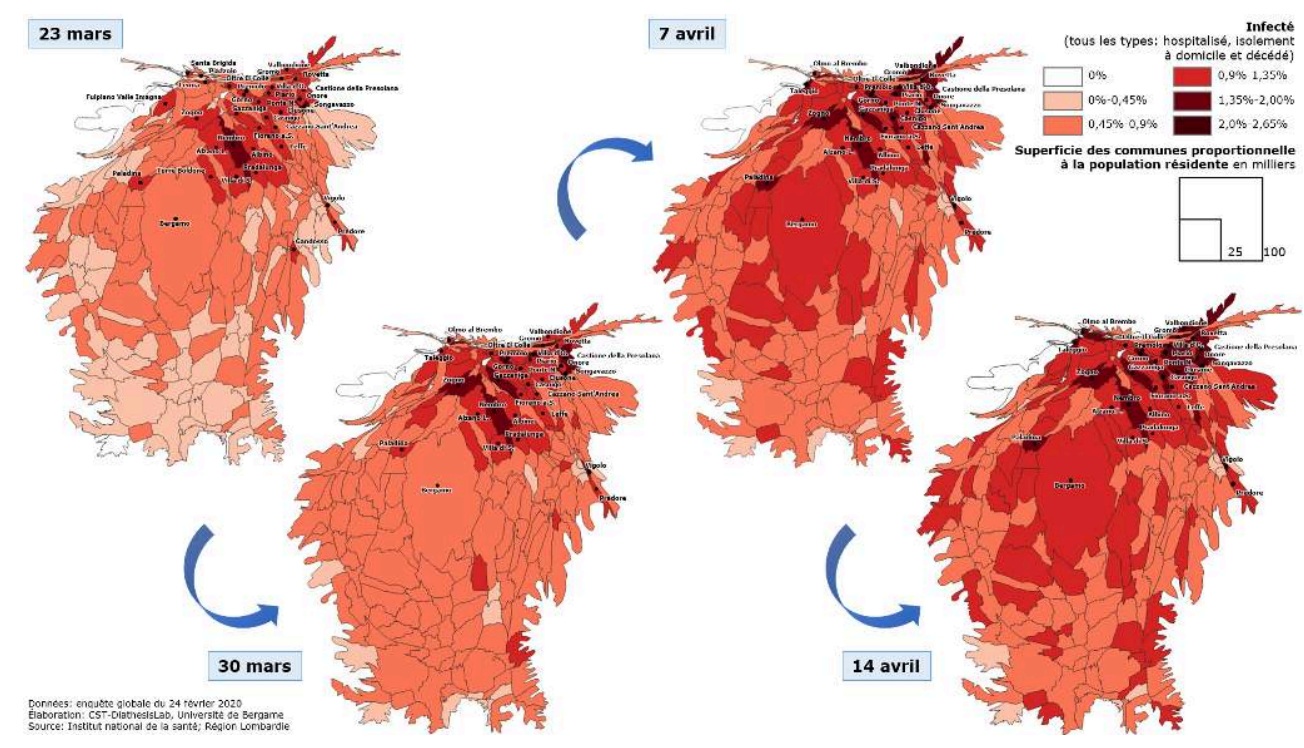

L'évolution présentée sur la figure 4 permet d'observer une augmentation progressive du nombre de contaminations au fil du temps. Dans un premier temps, la diffusion virale concerne principalement la Val Seriana, où l'on observe, ainsi que dans quelques communes de la Val Bembrana et de la Vallée Imagna, les taux de contamination les plus élevés de la province. La ville de Bergame, ainsi que diverses communes situées plus au sud et le reste des vallées, présentent un taux de contamination uniforme et plutôt contenu, tandis que la plaine bergamasque présente une diffusion relativement plus faible des contaminations. Dans un second temps, on peut noter que l'intensité des contaminations s'est étendue à toute la zone de plaine de la province méridionale, dans laquelle la quasi-totalité des communes enregistre un taux de contamination uniforme. On note enfin une intensification virale ultérieure, plus marquée dans la zone centrale de la plaine bergamasque, qui concerne la zone de Bergame et la couronne de communes qui l'entourent. On observe par ailleurs une aggravation de l'épidémie également dans les communes de montagne situées au nord de la province.

En ce qui concerne la Val Seriana, siège du foyer bergamasque, il apparaît que les communes de Nembro, Albino et Alzano Lombardo, situées dans la partie méridionale de la vallée, font partie des premières communes de la province à avoir enregistré un nombre élevé de cas, bien que la propagation de l'épidémie ait ensuite marqué le pas. À l'inverse, dans les communes centrales de la province (en particulier Bergame, Seriate, Dalmine et Treviglio), dans lesquelles l'épidémie s'est propagée ensuite, le nombre de personnes touchées par l'affection virale a augmenté progressivement ${ }^{22}$. Les informations que nous donne la représentation cartographique nous incitent donc à penser que, pour comprendre la diffusion du virus dans la province de Bergame, il est indispensable de tenir compte des données relatives au contexte socio-territorial afin de réfléchir aux dynamiques qui ont conduit à sa diffusion et à son intensification.

\section{Le foyer de la Val Seriana}

Le premier cas de contamination par la Covid-19 dans le Bergamasque a été constaté le 23 février 2020 à l'hôpital d'Alzano Lombardo, une commune de la Val Seriana. La 
vallée, située dans les Alpes bergamasques, se trouve au nord-est de l'aire urbaine de Bergame. Le territoire de la vallée suit le cours du fleuve Serio et présente un peuplement et un habitat diversifiés tant du point de vue administratif ${ }^{23}$ que fonctionnel. La Val Seriana inférieure (ou Basse vallée) regroupe 18 communes, dont plusieurs grandes villes, qui rassemblent $71,7 \%$ de la population de toute la vallée. On peut citer à titre d'exemple les communes d'Albino (qui compte plus de 18000 habitants), Alzano Lombardo (près de 14 000) et Nembro (environ 12 000), situées au fond de la vallée et dans lesquelles a été enregistré le plus grand nombre de cas. S'ajoutent à celles-ci les communes des vallées latérales, comme Gandino ou l'Altopiano di Selvino, dans lesquelles on a observé de nombreux cas d'infection à la Covid-19. À l'inverse, la Haute vallée (composée de 20 communes) est moins densément peuplée (on y trouve $28,3 \%$ de la population totale de la Val Seriana) mais c'est là que se situent la petite ville de Clusone et les centres de Castione della Presolana et de Rovetta, qui ont enregistré un nombre de contaminations élevé, toutefois dans une moindre mesure. La Val Seriana se présente comme une vallée fortement urbanisée et dense au sud, dans laquelle on observe la présence d'une conurbation. Sur les versants et sur les hauts-plateaux latéraux se trouvent au contraire des centres de moindre importance qui se caractérisent toutefois par une industrialisation élevée dans les secteurs textile et mécanique.

Figure 5. Distribution du nombre de cas de Covid-19 dans la Val Seriana

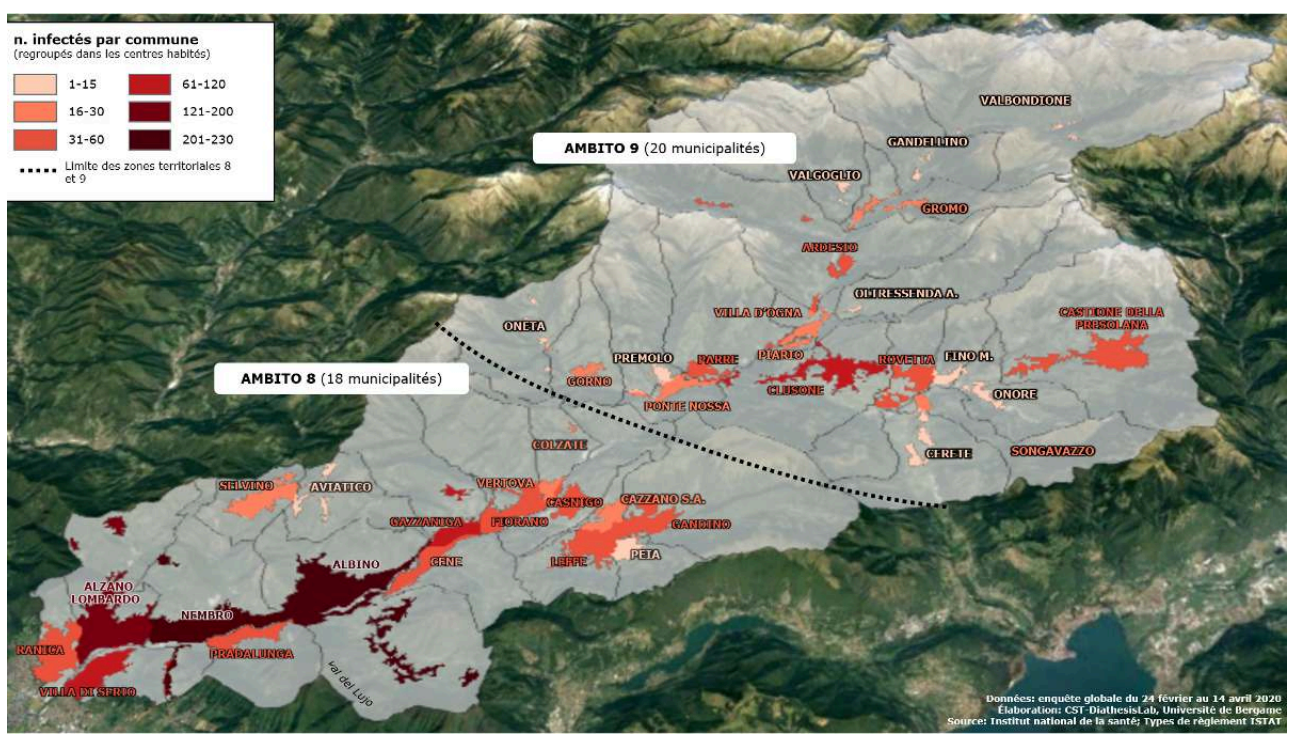

17 La carte illustre la situation de l'habitat en se servant des aspects morphologiques du territoire au moyen de la 3D, en référence au relief de la vallée ${ }^{24}$, tandis que les nuances de couleur traduisent la diffusion du virus au mois d'avril ${ }^{25}$ : Albino et Nembro sont les villes comptant le plus grand nombre de cas de Covid-19, suivies d'Alzano Lombardo. La Haute vallée semble quant à elle présenter une plus faible diffusion de l'épidémie, à l'exception du centre habité de Clusone. Cette situation pourrait être liée au plus faible nombre d'habitants ${ }^{26}$ et au fait que cette partie du territoire s'étend sur un hautplateau articulé, dans lequel la typologie de l'habitat, caractérisé par de petits centres et un habitat très dispersé, aurait pu favoriser la distanciation physique. Dans ce contexte, la mobilité et le déplacement des personnes pour motifs scolaires ou professionnels ont une importance toute particulière. 
Figure 6a. Mobilité pendulaire pour motifs scolaires dans la Val Seriana

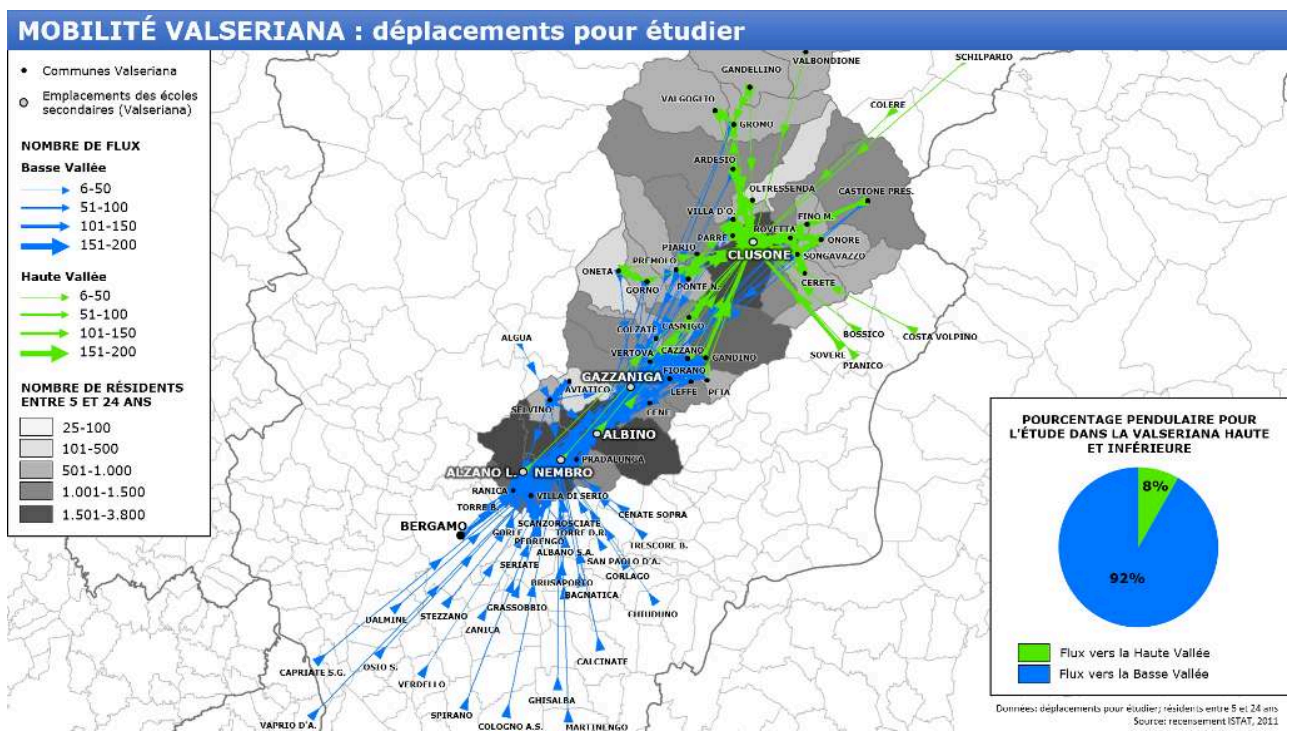

Figure 6b. Mobilité pendulaire pour motifs professionnels dans la Val Seriana

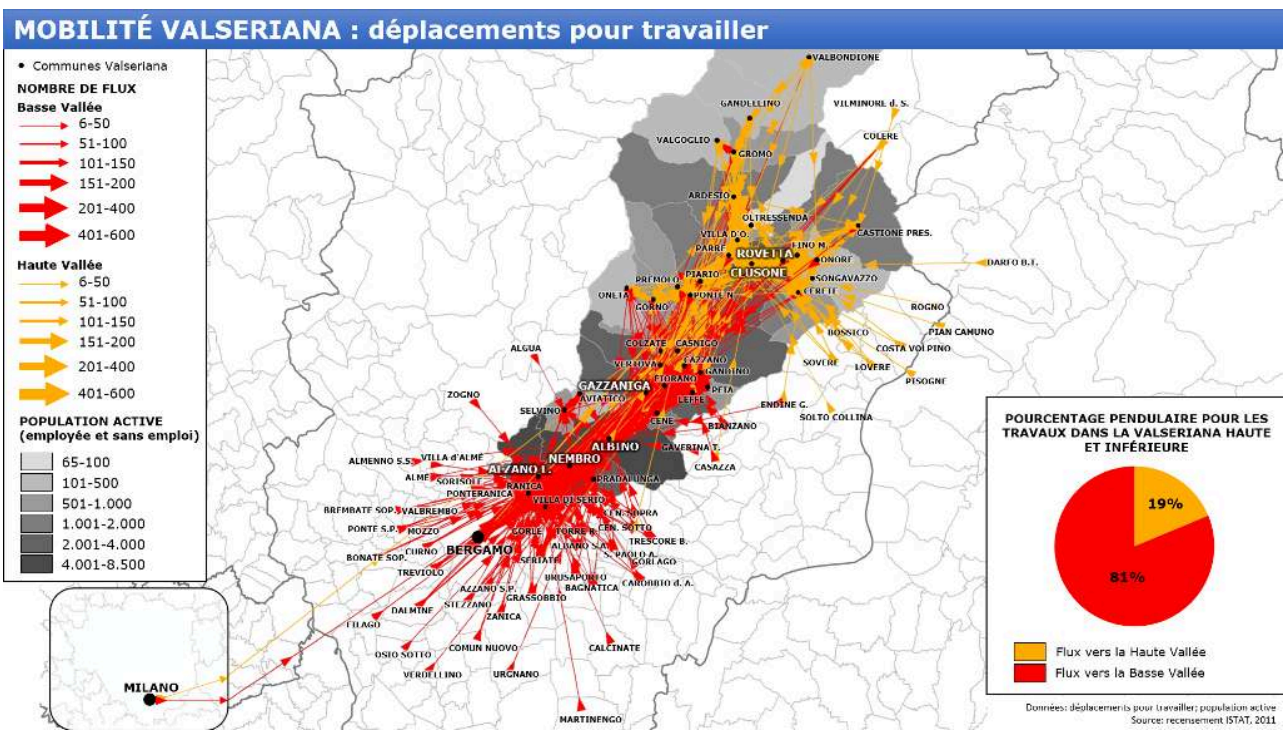

La cartographie relative à la mobilité scolaire représente le lieu de résidence des personnes qui se déplacent quotidiennement vers et depuis les principales communes dotées en établissements scolaires de la Val Seriana (Fig. 6a) ${ }^{27}$. Les établissements scolaires localisés dans les centres urbains de la conurbation de fond de vallée constituent des pôles d'attraction non seulement pour les élèves domiciliés dans la Val Seriana mais aussi pour ceux qui, bien que résidant à Bergame, dans les communes limitrophes de l'embouchure de la vallée ou dans la basse plaine bergamasque, se déplacent vers la Basse vallée pour raisons scolaires. Cette mobilité correspond à $92 \%$ des flux scolaires et se pose en nette contradiction avec les données relatives à la mobilité pendulaire pour la Haute vallée, qui ne représentent que $8 \%$ des déplacements pour motifs scolaires ${ }^{28}$. Les données relatives à la mobilité pendulaire pour motifs professionnels sont essentielles, surtout dans la Basse vallée ${ }^{29}$. Cette constance des flux s'explique par le nombre élevé d'entreprises et d'industries qui y sont implantées et qui 
emploient un nombre considérable de personnes ${ }^{30}$. La cartographie met en évidence la forte mobilité pendulaire interne à la Val Seriana et en particulier le fait que les travailleurs ${ }^{31}$ résidant dans les zones les plus isolées et sur les sites à flanc de montagne ou dans des zones limitrophes de la vallée se déplacent quotidiennement vers le fond de la vallée, autrement dit vers les principaux centres urbains et sièges d'entreprises. Par ailleurs, la cartographie met l'accent sur la mobilité pendulaire provenant de l'extérieur, et plus précisément des communes de la plaine voisine de la vallée et de la ville de Bergame ${ }^{32}$.

$19 \mathrm{Au}$ cœur de cette dynamique on trouve les mêmes communes que celles qui ont formé le foyer d'infection bergamasque ${ }^{33}$. Elles représentent en effet un point de passage obligé pour les territoires du nord, dans la mesure où la configuration particulière de la Val Seriana fait apparaitre un corridor de passage privilégié à l'intérieur duquel les voies de communication se révèlent particulièrement importantes. Il y a donc de bonnes raisons de penser que cette forte interconnexion territoriale a accentué les risques de contamination et, associée à d'autres facteurs concomitants tels que la défaillance du système de santé ${ }^{34}$, a joué un rôle considérable dans la propagation du virus.

\section{Interrogations et conclusions}

Menée à diverses échelles, l'étude de ce territoire, que l'épidémie a fortement et sévèrement frappé, a permis d'aborder en détail les facteurs géographiques susceptibles d'être considérés comme potentiellement liés à l'évolution de l'épidémie de Covid-19. Elle nous a apporté quelques éléments de réponse à la question de savoir pourquoi, à l'échelle de l'Italie, la pandémie a eu des effets dévastateurs sur certains territoires et plus légers sur d'autres. Dans le cadre de cette analyse, la Lombardie apparaît comme un terrain idéal pour la diffusion du coronavirus : la morphologie particulière de son territoire, la densité de son réseau d'échanges commerciaux nationaux et internationaux, la présence d'importants pôles industriels et d'entreprises sur le territoire, l'intensité de la mobilité pendulaire pour motifs scolaires et professionnels, et enfin la densité urbaine polycentrique ont favorisé une propagation intense du virus, avec la gravité qu'on connaît.

Le sens social du lieu a été démontré à travers l'utilisation d'outils cartographiques qui, en plus d'illustrer à plusieurs niveaux la signification du territoire et de faire apparaître la complexité de la pandémie actuelle, ont contribué à la compréhension du phénomène en mettant en évidence, en même temps, les fragilités territoriales qui ressortent de l'analyse.

Il serait imprudent de se servir de cette étude pour établir des généralités, même si sa démarche - à savoir la vision des modes de vie dans le monde contemporain mobile et urbanisé, qui se déploie à travers un entrelacs de nœuds et connexions produits par la dynamique de l'habitant - n'est pas spécifiquement lombarde ou italienne, mais peut s'appliquer à un grand nombre de régions du monde. Toutefois, à présent que certains aspects de ce mode de vie ont été incontestablement établis comme risqués ou "fragiles", cette approche devra être prise en compte dans la suite de cette « aventure » pandémique mondiale qui impose sa loi encore aujourd'hui. 


\section{BIBLIOGRAPHIE}

Ancidoni A., et al., 2020, Survey nazionale sul contagio COVID-19 nelle strutture residenziali e sociosanitarie. Istituto Superiore di Sanità, [en ligne], consulté le 25 novembre 2020, URL: https:// www.epicentro.iss.it/coronavirus/sars-cov-2-survey-rsa

Berloto S., Notarnicola N., Perobelli E., Rotolo A., 2020, Report on COVID-19 and Long-Term Care in Italy: lessons learned from an absent crisis management. LTC Responses to Covid-19, International Long-Term Care Policy Network, [en ligne], consulté le 25 novembre 2020, URL: https:// ltccovid.org/2020/04/10/report-on-covid-19-and-long-term-care-in-italy-lessons-learned-froman-absent-crisis-management/

Casti E., 1998, L'ordine del mondo e la sua rappresentazione. Semiosi e autoreferenza cartografica, Milano: Unicopli.

Casti E., 2013, Cartografia critica. Dal topos alla chora, Milano: Guerini Associati.

Casti E., 2020a, Geografia a 'vele spiegate': analisi territoriale e mapping riflessivo sul COVID-19 in Italia, Documenti Geografici, 1/2020, pp. 61-83.

Casti E., (dir.), 2020b, « Pourquoi Bergame ? Analyser le nombre de testés positifs au COVID-19 à l'aide de la cartographie. De la géolocalisation du phénomène à l'importance de sa dimension territoriale » Anthropocene2050, [en ligne], consulté le 25 août 2020, URL : https://medium.com/ anthropocene2050/pourquoi-bergame-analyser-le-nombre-de-test\%C3\%A9s-positifs-covid-19\%C3\%A0-laide-de-la-cartographie-7950cd0b452c.

Casti E., Adobati F., (dir.), (2020), Mapping riflessivo sul contagio del Covid-19. Dalla localizzazione del fenomeno all'importanza della sua dimensione territoriale. $3^{\circ}$ Rapporto di Ricerca, Le Tre Italie. Fragilità dell'abitare mobile e urbanizzato, URL: https://cst.unibg.it/it/ ricerca/progetti-ricerca (consulté le 25 août 2020), Bergamo: Università di Bergamo.

Cliff A.D., Haggett P., 2006, A swash-backwash model of the single epidemic wave. Journal of Geographical Systems, 8, n 3, pp. 227-252, DOI: 10.1007/s10109-006-0027-8.

Consolandi E., Rodeschini M., 2020, La cartografia come operatore simbolico: il contagio del Covid-19 in Lombardia. Documenti Geografici, 1/2020, pp. 711-724.

Conticini E., Frediani B., Caro D., 2020, Can atmospheric pollution be considered a co-factor in extremely high level of SARS-CoV-2 lethality in Northern Italy?, Environmental Pollution, 261, pp. $1-3$.

Cremaschi M, 2020, Pourquoi Bergame ? Le virus au bout du territoire, Métropolitiques, [en ligne], consulté le 24 août 2020, URL: https://www.metropolitiques.eu/Pourquoi-Bergame-Le-virus-aubout-du-territoire.html.

Djaïz D., 2020, « Coronavirus : la mondialisation malade de ses crises » Le Grand Continent, [en ligne], consulté le 25 août 2020, URL : https://legrandcontinent.eu/fr/2020/03/23/coronavirusmondialisation-david-djaiz/.

Fraser Taylor D.R., 2019, Cybercartography revisited, in Fraser Taylor D. R., Anonby E., Murasugi K., (dir), Further Developments in the Theory and Practice of Cybercartography, Amsterdam: Elsevier, pp. 3-23.

Gould P.R., 1969, Spatial Diffusion. Association of American Geographers. Washington: Association of American Geographers. 
Hagerstrand T., 1967, Innovation Diffusion as a Spatial Process, Chicago-London: The University of Chicago Press.

Jackson M.O., 2020, « Comment se diffuse un virus ?» Le Grand Continent, [en ligne], consulté le 25 août 2020, URL : https://legrandcontinent.eu/fr/2020/05/16/comment-se-diffuse-un-virus/

Kitchin R., 2020, Civil liberties or public health, or civil liberties and public health? Using surveillance technologies to tackle the spread of COVID-19. Space and Polity, https:// www.tandfonline.com/doi/full/10.1080/13562576.2020.1770587 (consulté le 25 août 2020).

ISTAT, (dir.), 2020, Rapporto annuale 2020. La situazione del Paese, Roma: Istituto Nazionale di Statistica, URL: https://www.istat.it/it/archivio/244848 (consulté le 25 août 2020).

ISTAT, Istituto Superiore di Sanità, (dir.), 2020, Impatto dell'epidemia Covid-19 sulla mortalità totale della popolazione residente. Primo trimestre 2020, Roma: Istituto nazionale di statistica e Istituto Superiore di Sanità URL: https://www.epicentro.iss.it/coronavirus/pdf/ Rapporto_Istat_ISS.pdf (consulté le 25 août 2020).

Lévy J., 1999, Le tournant géographique. Penser l'espace pour lire le monde, Parigi : Belin.

Lévy J., 2000, Les nouveaux espace de la mobilité, in Bonnet M., Desjeux D. (dir.), Les territoires de la mobilité, Parigi : Presses Universitaires de France, pp. 155-170.

Lévy J., (dir.), 2008, L'invention du monde. Une géographie de la mondialisation, Paris : Presses de Sciences Po (trad.: 2010, Inventare il mondo. Una geografia della mondializzazione, Londra: Pearson-Mondadori).

Lévy J., 2020, « L'humanité habite le Covid-19 » AOC. Analyse, Opinion, Critique, [en ligne], consulté le 25 août 2020, URL : https://aoc.media/analyse/2020/03/25/lhumanite-habite-le-covid-19/

Lussault M., 2003, lemma “Urbanité”, in Lévy J., Lussault M. (dir.), Dictionnaire de la Géographie, Parigi : Editions Belin, p. 966-967.

Lussault M., 2013, L'Avènement du Monde. Essai sur l'habitation humaine de la Terre, Paris : Seuil. Lussault M., 2017, Hyper-lieux. Les nouvelles géographies de la mondialisation, Paris : Seuil.

Lussault M., 2020, « Le Monde du virus - retourn sur l'eprouve de confinement » AOC. Analyse, Opinion, Critique, [en ligne], consulté le 25 août 2020, URL : https://aoc.media/analyse/ 2020/05/10/le-monde-du-virus-retour-sur-lepreuve-du-confinement/>

Mavagrani A., 2020, Tracking COVID-19 in Europe: Infodemiology Approach. JMIR Public Health Surveill, 6, n 2, URL: https://publichealth.jmir.org/2020/2/e18941/ (consulté le 24 août 2020).

Murgante B., Borruso G., Balletto G., Castiglia P., Dettori M., 2020, Why Italy First? Health, Geographical and Planning Aspects of the COVID-19 Outbreak. Sustainability, 12, n 5064, DOI: https:// doi.org/10.3390/su12125064.

Ogen Y., 2020, Assessing nitrogen dioxide (NO2) levels as a contributing factor to the coronavirus (COVID-19) fatality rate. Science of the Total Environment, 726, URL: https://www.ncbi.nlm.nih.gov/ pmc/articles/PMC7151460/pdf/main.pdf (consulté le 24 août 2020).

Söderström O., 2020, «Smart city citoyenne et pandémie » AOC. Analyse, Opinion, Critique, [en ligne], consulté le 25 août 2020, URL : https://aoc.media/analyse/2020/06/01/smart-citycitoyenne-et-pandemie/

Turco A., 1988, Verso una teoria geografica della complessità, Milano: Unicopli.

Zhou C., Su F., Pei T. et al., 2020, COVID-19: Challenges to GIS with Big Data. Geography and Sustainability, $1, \mathrm{n}^{\circ} 1$, pp. 77-87. 


\section{NOTES}

1. En s'appuyant sur l'acception épidémiologique de "foyer ", le terme désigne ici le lieu à partir duquel se diffuse l'agent pathogène, autour d'un groupe d'individus qui ont été soumis à une exposition virologique commune.

2. Pour de plus amples détails sur la recherche coordonnée par Emanuela Casti et Fulvio Adobati et intitulée >Mapping riflessivo sul contagio del Covid-19. Dalla localizzazione del fenomeno allimportanza della sua dimensione territoriale, voir : https://cst.unibg.it/it/ ricerca/progetti-ricerca (consulté le 25 août 2020).

3. L'expérience de l'évaluation de l'épidémie en Italie a soulevé des interrogations méthodologiques quant aux données mises à disposition par le ministère de la Santé et collectées par les Régions et leurs structures internes (notamment les services de santé régionaux). Ces données, qui contiennent des informations relatives au nombre absolu de cas de Covid-19 (à savoir le nombre de personnes hospitalisées, à l'isolement à domicile, ou décédées), ont été recueillies au moyen de méthodes de mesure différentes selon les territoires et sur la base de tests qui sont difficiles à interpréter et à comparer car ils n'ont pas été réalisés de façon uniforme sur l'ensemble de la population. Les données utilisées - en dépit de leur nature fragmentaire et hétérogène (Casti, 2020) sont issues de sources institutionnelles et proviennent notamment du Dipartimento di Protezione Civile - ministère de la Santé (http://opendatadpc.maps.arcgis.com/apps/ opsdashboard/index.html\#/b0c68bce2cce478eaac82fe38d4138b1), de la Région Lombardie (https://experience.arcgis.com/experience/ 0a5dfcc103d0468bbb6b14e713ec1e30/) et de l'Istituto Superiore di Sanità (https:// www.epicentro.iss.it/coronavirus/sars-cov-2-sorveglianza-dati). Certaines de ces données ont été rendues lisibles par machine grâce au travail de l'association OnData.

4. Voir à ce sujet les rapports publiés dans le cadre de la recherche Mapping riflessivo sul contagio del Covid-19. Dalla localizzazione del fenomeno all'importanza della sua dimensione territoriale, présente sur le site déjà cité.

5. En référence au rapport entre la propagation de la maladie et la mobilité on peut lire, dans le texte intitulé Rapporto annuale 2020, La situazione del Paese élaboré par l'Istituto nazionale di statistica, que «l'un des facteurs de la localisation et des différences d'intensité de propagation de l'épidémie sur le territoire est représenté par la mobilité professionnelle. L'analyse de la mortalité en référence à la géographie des systèmes locaux de travail (SL) permet de décrire l'impact de l'épidémie de Covid-19 à la lumière d'une caractérisation du territoire qui représente les systèmes urbains quotidiens, autrement dit les lieux dans lesquels les personnes travaillent et entretiennent l'essentiel de leurs relations sociales et économiques » (ISTAT, 2020, p. 86).

6. Pour les thèmes liés aux théories de la diffusion spatiale et épidémique, qui ne seront pas spécifiquement traités ici, voir Hägerstrand, 1967 et Gould, 1969. Le concept de diffusion en géographie est assurément un sujet largement étudié et recouvre "le déplacement d'un événement, ou d'un ensemble d'événements, donné dans l'espace et dans le temps, et se traduit par un processus et un modèle de répartition géographique " (Borruso et al., 2020, p.42). De même, les processus de diffusion épidémique ont été largement traités par Cliff et Haggett qui, dans le cadre de leurs travaux, expliquent comment de tels événements se produisent, à l'image de « vagues de diffusion spatiale » et trouvent leur origine dans un lieu précis (ou plusieurs lieux différents) pour ensuite s'étendre à 
des zones plus vastes sous l'effet d'interactions de diverse nature (Cliff \& Haggett, 2006).

7. D'après les géographes Jacques Lévy et Michel Lussault, à l'instar de la densité et de la diversité sociale, le degré d'urbanité est également lié à la configuration spatiale d'un territoire. De fait, la présence marquée d'espaces publics à forte densité de population contribue à la définition d'une entité urbaine. Pour plus de détails, voir : Lussault, 2003, pp. 966-967.

8. Sur la complexité et les implications d'un tel habitat, et sur les dynamiques de propagation du virus dans ce contexte, voir: Lévy, 1999, 2020; Lussault, 2017, 2020; Djaïz, 2020; Jackson, 2020.

9. Pour plus de détails sur le rôle de la cartographie comme opérateur symbolique et sur la contribution de la cartographie à la compréhension de l'infection par la Covid-19 en Lombardie, voir : Consolandi \& Rodeschini, 2020.

10. La Lombardie se trouve au centre de la plaine du Pô, une région caractérisée par un climat continental tempéré ainsi que par une morphologie particulière. La plaine du Pô se présente en effet comme un vaste bassin de forme plane fermé sur trois côtés, ce qui contribue à favoriser la persistance au sol des émissions polluantes susceptibles d'avoir facilité la diffusion de la pandémie (Murgante et al., 2020, Ogen, 2020).

11. Si l'on tient compte toutefois de l'indice de vieillissement calculé par l'institut de la statistique (Istat), il est intéressant de noter que l'on trouve parmi les régions dans lesquelles la proportion de personnes âgées est supérieure à la moyenne nationale essentiellement la Ligurie, le Molise, le Frioul-Vénétie Julienne, la Sardaigne, le Piémont et la Toscane. Inversement, les régions dans lesquelles l'indice de vieillissement est le plus bas sont notamment la Campanie, suivie du Trentin-HautAdige, de la Sicile, du Latium, de la Calabre et de la Lombardie. Cf. : Casti \& Adobati, 2020. Les données relatives au faible taux de natalité ou à l'espérance de vie en Italie sont consultables sur les sites : http://demo.istat.it/; https://www.istat.it/it/archivio/ 238447 (consulté le 25 octobre 2020).

12. L'anamorphose est une technique imaginée à la Renaissance dans le but de déformer les figures. Elle consiste à déformer délibérément l'image que l'on veut représenter afin de "mettre en lumière le fait que l'image ne permet pas de restituer la signification des choses et, par voie de conséquence, afin d'avertir qu'il ne faut pas se fier aveuglément aux apparences » (Casti, 2013, p. 276).

13. Jacques Lévy indique que les aires périurbaines apparaissent comme les premiers centres à avoir été touchés par la pandémie et souligne, plus précisément, que «les plus grandes densités de cas se rencontrent plutôt dans des villes petites et moyennes [...], dans des situations où ce ne sont pas les liens faibles typiques des grandes villes qui dominent et où les interactions supposant une interconnaissance représentent une grande part de l'ensemble des liens » (Lévy, 2020).

14. On observe sur la carte que certaines communes affichent un taux élevé de contamination, alors qu'elles ne sont pas situées dans les provinces de Lodi, Crémone, Bergame et Mantoue.

15. Le nombre de décès enregistrés au mois de mars et avril 2020 dans les provinces citées est jusqu'à quatre fois supérieur aux taux de mortalité enregistrés à la même époque les années précédentes (Casti \& Adobati, 2020, pp. 39-46). 
16. La vitesse de diffusion de la pandémie et son intensité en Lombardie ont été comparées au système de soins régional et rapportées au nombre de personnes âgées vivant en résidences pour personnes âgées présentes sur le territoire. Voir : Casti \& Adobati, 2020.

17. Elle se situe dans la partie centre-orientale de la Lombardie, dont les limites sont marquées par la chaîne des Alpes bergamasques et par ses bassins hydrographiques, en particulier ceux du Serio, du Brembo, de l'Adda et de l'oglio. Ces rivières, qui sont d'importants cours d'eau, matérialisent aussi les limites de la province.

18. L'aéroport international "Il Caravaggio" de Bergame-Orio al Serio a connu ces dernières années une croissance exponentielle de son trafic, due à son offre de mobilité low-cost. En 2019, l'aéroport de Bergame-Orio al Serio a accueilli 13857257 passagers, un chiffre qui le classe parmi les plus grands aéroports du pays (Données Assoaeroporti, http://www.assaeroporti.com/, consulté le 25 août 2020). S'ajoute à ce constat une mobilité pendulaire intense entre les divers pôles urbains tels Bergame, Brescia et Milan qui, en plus de regrouper les sièges des institutions du territoire, voient s'implanter de grandes entreprises ainsi que des universités et organismes de recherche d'envergure nationale et internationale. Ces dernières années, son attractivité progresse également auprès des touristes, au point que Bergame n'est plus simplement une escale mais une destination touristique à part entière.

19. Rappelons simplement qu'au moment de l'entrée en vigueur du décret du Président du Conseil des ministres (DPCM) du 8 mars portant Mesures urgentes de confinement et de gestion de l'urgence épidémiologique Covid-19, qui prévoyait l'application du confinement en Lombardie et dans 14 autres provinces du nord de l'Italie, la province de Bergame comptait déjà 997 cas d'infection à la Covid-19. Ces chiffres avaient encore grimpé en flèche deux jours seulement après l'entrée en vigueur du décret. Ainsi, le 10 mars (date à laquelle a été publié le DPCM Mesures supplémentaires de confinement et de gestion de l'urgence épidémiologique Covid-19 sur tout le territoire national - aussi appelé \#iorestoacasa (restez chez vous) -, qui étend à l'ensemble du territoire national les mesures de confinement et les dispositions déjà prévues dans le décret cité précédemment), la province de Bergame enregistrait 1472 cas de Covid-19 et devenait la province d'Italie présentant le plus grand nombre de cas. Pour plus de détails sur l'évolution nationale $d u$ nombre de cas en Italie, cf.: http:// opendatadpc.maps.arcgis.com/apps/opsdashboard/index.html\#/

b0c68bce2cce478eaac82fe38d4138b1 (consulté le 25 août 2020).

20. Pour l'élaboration de ces cartes nous avons utilisé les données fournies par l'Istituto Superiore di Sanità et la Région Lombardie relatives au nombre de cas par commune (décès, patients en réanimation, patients symptomatiques, isolements à domicile, retours à domicile) et réactualisées - suivant l'évolution de l'épidémie - jusqu'au 14 avril 2020 (https://www.epicentro.iss.it/coronavirus/sars-cov-2-sorveglianza-dati). Les communes apparaissant en blanc sur la carte ont un nombre de contaminations égal à zéro, ce qui concerne également les communes pour lesquelles on ne dispose pas du nombre de cas.

21. Il est question ici de l'approche théorique issue des études sur la complexité territoriale présentée par Raffestin puis traitée par Turco, qui considère la géographie comme une forme territoriale de l'action sociale. Pour plus de détails, voir Turco, 1988.

22. Cf., pour preuve, les rapports régionaux produits par les systèmes de surveillance hebdomadaire du ministère de la Santé: http://www.salute.gov.it/portale/ 
nuovocoronavirus/dettaglioNotizieNuovoCoronavirus.jsp?lingua=italiano\&id=5025 (consulté le 25 août 2020).

23. Cette subdivision correspond aux zones territoriales représentées, à savoir la zone (Ambito) $n^{\circ} 8$ correspond à la Basse Val Seriana et la zone $n^{\circ} 9$ à la Haute vallée. Plus précisément, la zone territoriale (Ambito) $n^{\circ} 8$ de Val Seriana a pour centre Albino et comprend 18 communes. La zone $n^{\circ} 9$, quant à elle, bien qu'étant la moins peuplée, comprend 20 communes et se subdivise en trois sous-zones distinctes : i) Zone $\mathrm{n}^{\circ} 9$ Réunion des communes de la Presolana et de Clusone ; ii) Zone $n^{\circ} 9$ - Asta del Serio ; iii) Zone $\mathrm{n}^{\circ} 9$ - Val Dossana et del Riso.

24. Il faut rappeler ici l'importance de la cartographie de paysage qui, à travers la figuration en perspective, intègre les valeurs et les connaissances que la société attribue à son territoire, en en faisant ressortir la complexité (Casti, 2013, pp. 103-106).

25. L'utilisation de cette méthodologie représentative permet notamment une distribution non uniforme des données sur toute la superficie de la commune, mais limite la figuration de la diffusion effective du nombre de cas aux centres habités. Les données fournies par l'Institut supérieur de la santé et la Région Lombardie portent sur le nombre de cas par commune mis à jour au 14 avril 2020 (https:// www.epicentro.iss.it/coronavirus/sars-cov-2-sorveglianza-dati).

26. Rappelons que la conurbation de la Basse vallée compte près de 100000 habitants et représente $71,1 \%$ de la population totale de la Val Seriana, tandis que 40000 personnes résident dans la Haute vallée, soit les $28,3 \%$ restants de la population.

27. Il n'a malheureusement pas été possible d'effectuer la cartographie de la mobilité pendulaire universitaire, qui aurait sans nul doute considérablement nourri la réflexion.

28. Les écoles situées dans la vallée septentrionale sont essentiellement fréquentées par des élèves résidant dans la Haute Val Seriana et attirent dans une moindre mesure les élèves en provenance des vallées voisines, telles que la Val di Scave, et de quelques communes limitrophes plus au sud.

29. La mobilité pendulaire dans la Basse vallée est marquée par les déplacements quotidiens de près de 26000 travailleurs, représentant $81 \%$ des déplacements pour motifs professionnels. Inversement, la mobilité professionnelle vers la Haute vallée représente $19 \%$ des flux.

30. Ainsi, rien qu'entre Nembro et Alzano, des communes situées à l'embouchure de la vallée, on compte 376 entreprises, qui emploient près de 4000 salariés. Le système productif de la Val Seriana semble en effet solide et fortement interconnecté avec le territoire à l'échelle nationale et internationale. D'après le registre statistique des unités locales (ISTAT), il ressort spécifiquement que le bas de la Val Seriana regroupe principalement des activités professionnelles employant moins de 50 salariés. En outre, on observe une présence massive d'activités liées au bâtiment, au commerce et à la réparation de véhicules, suivies des fonctions professionnelles, scientifiques et techniques (qui incluent, entre autres, les fonctions liées au conseil juridique et à la comptabilité, ou à l'architecture) et des activités manufacturières. Voir: https:// www.bg.camcom.it/; http://dati.istat.it/index.aspx?queryid=21145 (consultés le 25 novembre 2020).

31. En référence à la population active résidant dans les communes de la Val Seriana, il faut souligner la dichotomie entre la Basse vallée, densément peuplée et qui compte 
une main-d'œuvre plus nombreuse, et la Haute vallée, dans laquelle la population résidente compte moins d'actifs. La population active est classée par l'ISTAT conformément aux définitions d'Eurostat et est subdivisée entre les personnes exerçant une activité et les personnes sans emploi (ou à la recherche d'un emploi), de plus de 15 ans. Voir: https://www.istat.it/it/metodi-e-strumenti/glossario (consulté le 25 août 2020).

32. Dans ce contexte, la présence de la ligne de tramway appelée "Tram delle Valli » (ou T1), qui relie le centre urbain de Bergame et la ville d'Albino, est particulièrement importante. C'est une ligne interurbaine qui dessert justement la ville de Bergame et la Val Seriana inférieure.

33. Il convient toutefois de préciser qu'à la fois le territoire de Lodi (et en particulier les communes de Codogno et (asalpusterlengo) et celui de la Val Seriana sont des zones à vocation industrielle marquée, notamment pour le secteur de la production et de la logistique. Ces deux territoires bénéficient de la proximité avec deux artères routières importantes à l'échelle du pays et à l'échelle européenne, à savoir l'autoroute A1 MilanNaples et l'autoroute A4 Turin-Trieste. Du point de vue du nombre de cas, ces deux zones ont été fortement touchées par l'épidémie de Covid-19. Malgré cela, si le confinement a été décidé par le gouvernement italien dans la province de Lodi, en application du décret-loi $\mathrm{n}^{\circ} 6$ du 23 février 2020 portant Mesures urgentes de confinement et de gestion de l'urgence épidémiologique Covid-19, il a fallu attendre jusqu'au 8 mars pour que les mesures de restriction soient étendues à l'ensemble de la Lombardie. Certaines des causes qui expliquent la diffusion rapide de la Covid-19 en Lombardie peuvent donc être liées aux politiques de gestion de l'épidémie au niveau national et/ou local ainsi qu'à la lenteur de mise en œuvre des mesures pour circonscrire les zones les plus sévèrement touchées par le virus.

34. Il est important de souligner que le système de santé (en particulier l'hôpital d'Alzano Lombardo) et le classement trop tardif en zone rouge des communes de Nembro et d'Alzano Lombardo ont été des facteurs particulièrement nets de la diffusion du virus dans la Val Seriana et sur tout le territoire de la province en général. Cette question a été portée devant les juridictions pénales et fait actuellement l'objet d'une évaluation de la part des autorités judiciaires.

\section{RÉSUMÉS}

La Lombardie est tristement connue pour avoir été la région italienne la plus lourdement touchée par le virus SARS-CoV-2. Les premiers grands foyers italiens de la pandémie ont en effet été identifiés dans cette région, plus particulièrement dans les provinces de Lodi, Crémone et Bergame (et plus précisément dans la Val Seriana). Pour mieux comprendre l'intensité et la gravité de l'impact de l'épidémie en Lombardie et notamment dans la province de Bergame, un groupe de chercheurs du CST-Centro Studi sul Territorio de l'Université de Bergame a mené une étude afin de déterminer si les causes de cette épidémie pouvaient être liées aux aspects sociaux du territoire. Cette analyse a donc pour but de comprendre si la complexité des structures résidentielles et fonctionnelle du territoire a pu aggraver les effets de l'infection sur la 
population de Lombardie, notamment en raison de sa diffusion rapide à partir des premiers foyers de propagation dans la Val Seriana.

Les premières données relatives aux principaux foyers de la pandémie ont conduit tout d'abord à supposer que la propagation du virus s'expliquait par le réseau de connexions étroit entre ces différents lieux. Dans un second temps, c'est le facteur de la proximité qui pourrait justifier la propagation du virus, car les municipalités les plus touchées font partie de la conurbation multicentrique qui caractérise la région Lombardie et la plaine du Pô de manière générale.

\section{INDEX}

Mots-clés : analyse territoriale, cartographie réflexive, Covid-19

Index géographique : Italie, Lombardie, Val Seriana

\section{AUTEUR}

\section{ELISA CONSOLANDI}

Doctorante à l'Université de Bergame 